\title{
Observational Constraints of 30-40 GeV Dark Matter Annihilation in Galaxy Clusters
}

\author{
Man Ho Chan \\ The Hong Kong Institute of Education, Tai Po, Hong Kong \\ Correspondence should be addressed to Man Ho Chan; chanmh@ied.edu.hk
}

Received 5 October 2015; Accepted 6 January 2016

Academic Editor: W. Niel Brandt

Copyright (C) 2016 Man Ho Chan. This is an open access article distributed under the Creative Commons Attribution License, which permits unrestricted use, distribution, and reproduction in any medium, provided the original work is properly cited.

\begin{abstract}
Recently, it has been shown that the annihilation of $30-40 \mathrm{GeV}$ dark matter particles through $b \bar{b}$ channel can satisfactorily explain the excess GeV gamma-ray spectrum near the Galactic Center. In this paper, we apply the above model to galaxy clusters and use the latest upper limits of gamma-ray flux derived from Fermi-LAT data to obtain an upper bound of the annihilation cross section of dark matter. By considering the extended density profiles and the cosmic ray profile models of 49 galaxy clusters, the upper bound of the annihilation cross section can be further tightened to $\langle\sigma v\rangle \leq 9 \times 10^{-26} \mathrm{~cm}^{3} \mathrm{~s}^{-1}$. This result is consistent with the one obtained from the data near the Galactic Center.
\end{abstract}

\section{Introduction}

In the past few decades, high energy gamma-ray near the Galactic Center was detected. The origin of this gammaray is commonly believed to be the cosmic ray due to high energy proton-proton collisions and emission from pulsars $[1,2]$. However, recently, Hooper et al. [3] point out that a large diffuse signal of gamma-ray is obtained near the Galactic Center, which is hard to be explained by the cosmic ray and pulsar emission. In particular, even including both known sources and unidentified sources, the millisecond pulsars can only account for no more than 10 percent of the $\mathrm{GeV}$ excess [3]. Although recent studies report some evidence for unresolved point sources in the inner galaxy which can explain part of the excess gamma-ray signal $[4,5]$, the possibility of the emission of gamma-ray due to the dark matter annihilation is still a very popular model to explain the observed diffuse signal $[1,2,6-8]$. Moreover, Daylan et al. [6] discover that the gamma-ray spectrum obtained from Fermi-LAT data can be well fitted with $b \bar{b}$ annihilation channel of dark matter particles. The required rest mass of the dark matter particle is about $m_{\chi} \approx 30-40 \mathrm{GeV}$ and the annihilation cross sections obtained by two different groups are $\langle\sigma v\rangle=(1.4-2.0) \times 10^{-26} \mathrm{~cm}^{3} \mathrm{~s}^{-1}[6]$ and $\langle\sigma v\rangle=$ $(2.7-7.5) \times 10^{-26} \mathrm{~cm}^{3} \mathrm{~s}^{-1}[1]$, respectively. The obtained cross sections are consistent with the expected canonical thermal relic abundance cross section $\left(\langle\sigma v\rangle \approx 3 \times 10^{-26} \mathrm{~cm}^{3} \mathrm{~s}^{-1}\right)$ in cosmology. Furthermore, Moore et al. (1999) point out that the inner slope of the radial-dependence of the gamma-ray emission is $\gamma \approx 1.1-1.3$ (the best fit is $\gamma=1.26$ ), which is consistent with the numerical simulation of dark matter halo structure $\gamma=1-1.5[9,10]$.

Besides the emission of gamma-ray near the Galactic Centre, Fermi-LAT also reports the emission of gamma-ray from different galaxy clusters. Although most galaxy clusters are located far away from us, the dark matter annihilation signals from some of them are still significant because they have a larger amount of dark matter. Therefore, it is worthwhile to detect gamma-ray flux emitted by some nearby galaxy clusters. The first systematic study of 33 galaxy clusters by Fermi-LAT obtained some upper limits on the gamma-ray flux in the range $0.2-100 \mathrm{GeV}$. The typical values of the flux are about $(1-5) \times 10^{-9} \mathrm{ph} \mathrm{cm}^{-2} \mathrm{~s}^{-1}$ [11]. However, these upper limits are too high to constrain the flux due to the annihilation of dark matter, which is of the order $10^{-10} \mathrm{ph} \mathrm{cm}^{-2} \mathrm{~s}^{-1}$. Later, Ando and Nagai [12] start to realize that the gamma-ray flux data from Fornax cluster is able to constrain the annihilation dark matter model because the cosmic ray emission from Fornax cluster does not dominate the gamma-ray emission. By using their data, the cross section constrained from 


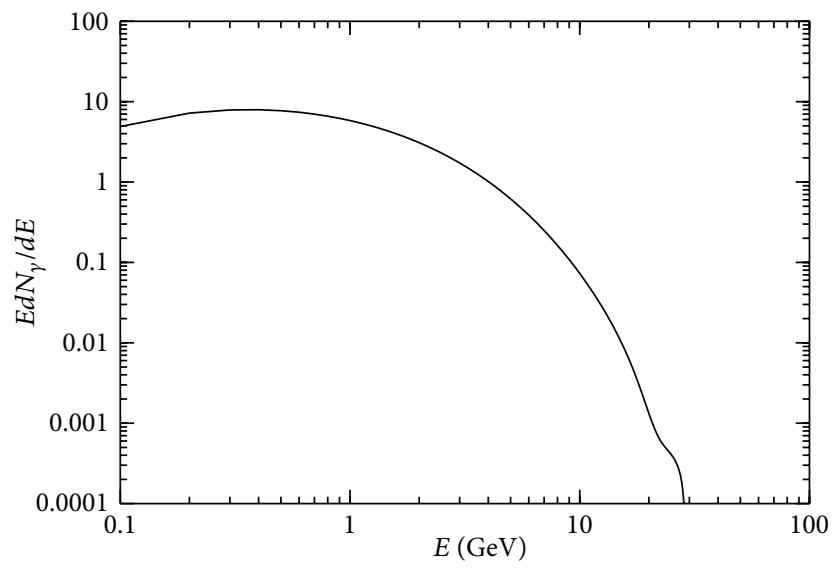

FIGURE 1: The energy spectrum of photons emitted per one annihilation $E d N_{\gamma} / d E$ for $m_{\chi}=30 \mathrm{GeV}$.

the Fornax cluster is $\langle\sigma v\rangle \leq(2-3) \times 10^{-25} \mathrm{~cm}^{3} \mathrm{~s}^{-1}$ [12]. This upper limit, however, is a factor of 5 greater than that obtained from the data near the Galactic Center.

Fortunately, the recent observations of 50 galaxy clusters in 4 years of Fermi-LAT data narrow down the upper flux limit to the order of $10^{-10} \mathrm{ph} \mathrm{cm}^{-2} \mathrm{~s}^{-1}$, which may provide a better constraint on the dark matter annihilation [13]. In this paper, we calculate the latest constraint on the dark matter annihilation cross section through $b \bar{b}$ channel based on these recent observations. We also realize that there are 2 galaxy clusters (Fornax and A2877) which are good candidates to constrain the properties of dark matter annihilation.

\section{Dark Matter Annihilation in Galaxy Clusters}

The total number of photons with energy greater than $E_{0}$ produced by the annihilation of dark matter within a galaxy cluster can be calculated by

$$
\dot{N}_{\gamma}=4 \pi \int_{0}^{R_{200}} \rho_{\mathrm{DM}}^{2}\langle\sigma v\rangle m_{\chi}^{-2} r^{2} d r \int_{E_{0}}^{\infty} \frac{d N_{\gamma}}{d E} d E,
$$

where $\rho_{\mathrm{DM}}$ is the dark matter density profile, $R_{200}$ is the effective radius of a galaxy cluster, and $d N_{\gamma} / d E$ is the energy spectrum of gamma-ray produced per one annihilation. The final-state spectrum $E d N_{\gamma} / d E$ can be generated by PYTHIA simulations. It can be well fitted by the following analytic formula (see Figure 1) [16]:

$$
\begin{aligned}
x^{1.5} \frac{d N_{\gamma}}{d x}= & a_{1} \exp \left(-b_{1} x^{n_{1}}-b_{2} x^{n_{2}}-\frac{C_{1}}{x^{d_{1}}}+\frac{C_{2}}{x^{d_{2}}}\right) \\
& +q x^{1.5} \ln [p(1-x)] \frac{x^{2}-2 x+2}{x},
\end{aligned}
$$

where $x=E / m_{\chi}$ and $a_{1}, b_{1}, b_{2}, n_{1}, n_{2}, C_{1}, C_{2}, d_{1}, d_{2}$, $q$, and $p$ are all fitted parameters. Some of the parameters depend on $m_{\chi}$ and they can be estimated by using simple power law in $m_{\chi}$ [16]. For $m_{\chi}=30-40 \mathrm{GeV}$, the values $\Phi \equiv m_{\chi}^{-2} \int\left(d N_{\gamma} / d E\right) d E$ are equal to $(0.030 \pm 0.001) \mathrm{GeV}^{-2}$

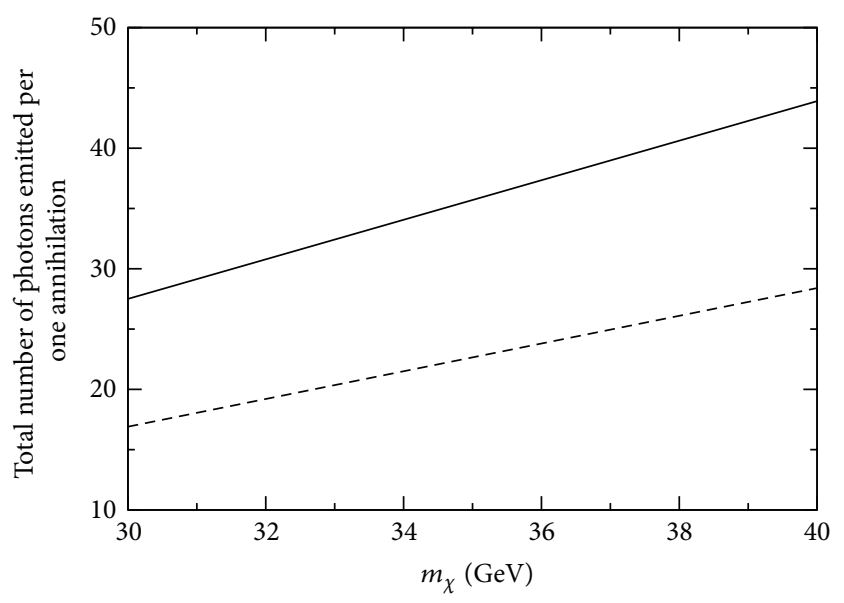

FIgURE 2: The total number of photons emitted per one annihilation $\int\left(d N_{\gamma} / d E\right) d E$ for $E_{0} \geq 500 \mathrm{MeV}$ (solid line) and $E_{0} \geq 1 \mathrm{GeV}$ (dashed line) versus $m_{\chi}$.

and $(0.018 \pm 0.001) \mathrm{GeV}^{-2}$ for $E_{0}=500 \mathrm{MeV}$ and $E_{0}=1$ $\mathrm{GeV}$, respectively (see Figure 2). The reason of using these two energy bins $\left(E_{0}=500 \mathrm{MeV}\right.$ and $\left.1 \mathrm{GeV}\right)$ is to match the observed fluxes in [13]. Since the values of $\Phi$ vary less than $4 \%$ of the mean value, for simplicity, we just assume that this value is a constant in the following discussion.

We apply the NFW density profile to model the mass density profile of dark matter in galaxy clusters [10]:

$$
\rho_{\mathrm{DM}}=\frac{\rho_{s} r_{s}^{3}}{r\left(r+r_{s}\right)^{2}}
$$

where $\rho_{s}$ and $r_{s}$ are the scale density and scale radius of a galaxy cluster, respectively. By using the virial mass $M_{200}$, we can get the concentration parameter $c$ for each galaxy cluster by a universal scaling relation [17]. Therefore, we can get the scale density $\rho_{s}$ and $r_{s}=r_{200} / c$ for each galaxy cluster. The observed gamma-ray flux from the above extended profile within a solid angle $\Delta \Omega$ along the line of sight can be calculated by

$$
\begin{aligned}
\phi(\Delta \Omega) & =\frac{1}{8 \pi} \frac{\langle\sigma v\rangle}{m_{\chi}^{2}} \int_{E_{0}}^{\infty} \frac{d N_{\gamma}}{d E} d E \int_{\Delta \Omega} d \Omega \int_{\mathrm{los}} \rho_{\mathrm{DM}}^{2} d s \\
& =\frac{\Phi}{8 \pi}\langle\sigma v\rangle J_{\mathrm{NFW}} .
\end{aligned}
$$

In general, the $J$-factor $J_{\mathrm{NFW}}$ depends on $\rho_{s}, r_{s}$, and the size and the distance of a galaxy cluster.

\section{Comparing the Gamma-Ray Flux with the Observed Data}

Since most of the galaxy clusters are extended sources, we compare the calculated flux from (4) with the upper limit of the "extended flux" obtained from Fermi-LAT [13]. The $J$-factor (in $\mathrm{GeV}^{2} \mathrm{~cm}^{-5}$ ) calculated by using the angle of view measured is about $\log \left(J_{\mathrm{NFW}}\right) \approx 18$ (see Table 1 ), which is 
TABLE 1: The scale radius (in $\mathrm{Mpc}$ ), the scale density (in $\left.10^{14} M_{\odot} \mathrm{Mpc}^{-3}\right), J$-factor (in $\log \left(\mathrm{GeV}^{2} \mathrm{~cm}^{-5}\right)$ ), the gamma-ray flux from cosmic ray emission $\Phi_{\mathrm{CR}}$ [13-15], the observed gamma-ray flux upper limits $(95 \% \mathrm{CL}) \Phi_{\mathrm{UL}}[13]$, and the calculated upper limits (95\% $\mathrm{CL})$ of the annihilation cross section $\left(\langle\sigma v\rangle_{1}^{\mathrm{UL}}\right.$ and $\left.\langle\sigma v\rangle_{2}^{\mathrm{UL}}\right)$ for A2877 and Fornax. All the units for the gamma-ray flux and annihilation cross section are in $10^{-10} \mathrm{~cm}^{-2} \mathrm{~s}^{-1}$ and $10^{-26} \mathrm{~cm}^{3} \mathrm{~s}^{-1}$, respectively.

\begin{tabular}{lcc}
\hline & A2877 & Fornax \\
\hline$r_{s}$ & 0.411 & 0.196 \\
$\rho_{s}$ & 8.67 & 12.9 \\
$J_{\mathrm{NFW}}$ & 17.3 & 18.1 \\
$\Phi_{\mathrm{CR}}(E \geq 500 \mathrm{MeV})$ & 0.51 & 0.84 \\
$\Phi_{\mathrm{CR}}(E \geq 1 \mathrm{GeV})$ & 0.29 & 0.49 \\
$\Phi_{\mathrm{UL}}(E \geq 500 \mathrm{MeV})$ & 0.9 & 3.1 \\
$\Phi_{\mathrm{UL}}(E \geq 1 \mathrm{GeV})$ & 0.43 & 1.4 \\
$\Phi_{\mathrm{DM}}(E \geq 500 \mathrm{MeV})$ & 0.39 & 2.3 \\
$\Phi_{\mathrm{DM}}(E \geq 1 \mathrm{GeV})$ & 0.94 & 0.14 \\
$\langle\sigma v\rangle_{1}^{\mathrm{UL}}(E \geq 500 \mathrm{MeV})$ & 37 & 19 \\
$\langle\sigma v\rangle_{1}^{\mathrm{UL}}(E \geq 1 \mathrm{GeV})$ & 28 & 14 \\
$\langle\sigma v\rangle_{2}^{\mathrm{UL}}(E \geq 500 \mathrm{MeV})$ & 16 & 14 \\
$\langle\sigma v\rangle_{2}^{\mathrm{UL}}(E \geq 1 \mathrm{GeV})$ & 9.2 & 9.0 \\
\hline
\end{tabular}

consistent with the result obtained previously [18]. In order to match the observational data, we examine $E_{0}=500 \mathrm{MeV}$ and $E_{0}=1 \mathrm{GeV}$ [13]. By assuming $\langle\sigma v\rangle=10^{26} \mathrm{~cm}^{3} \mathrm{~s}^{-1}$, the ranges of the flux calculated by (4) based on the sample in Ackermann et al. [13] (49 galaxy clusters) are (0.001-0.17) $\times 10^{-10} \mathrm{ph} \mathrm{cm}^{-2} \mathrm{~s}^{-1}$ for $E_{0}=500 \mathrm{MeV}$ and $(0.007-1.0) \times 10^{-11} \mathrm{ph} \mathrm{cm}^{-2} \mathrm{~s}^{-1}$ for $E_{0}=1 \mathrm{GeV}$, respectively. The obtained ranges of flux are much smaller than the upper limits obtained by Fermi-LAT data for many galaxy clusters except 2 nearby galaxy clusters (A2877 and Fornax) whose gamma-ray flux upper limits (95\% CL) are close to their corresponding annihilation gamma-ray flux. If we assume that all gamma-ray flux is due to the dark matter annihilation, we can obtain an upper limit of annihilation cross section $\langle\sigma v\rangle_{1}^{\mathrm{UL}}$ for each galaxy cluster (see Table 1). In particular, the Fornax cluster gives the tightest upper bound (95\% CL) for the annihilation cross section $\langle\sigma v\rangle \leq 1.9 \times 10^{-25} \mathrm{~cm}^{3} \mathrm{~s}^{-1}$, which is just a little bit tightened compared with the result obtained by Ando and Nagai [12].

Nevertheless, in most galaxy clusters, the major contribution of the gamma-ray flux is the cosmic ray emission due to proton-proton collisions [11]. Recently, Pinzke and Pfrommer [14] discover a universal scaling relation to model the cosmic ray emission in galaxy clusters. Later, Pinzke et al. [15] give a better model to estimate the gamma-ray contribution from cosmic ray. If we assume that some of the gamma-ray flux is contributed by the cosmic ray emission, and the remaining flux is due to the dark matter annihilation, we can obtain a tighter constraint on the upper bound of the annihilation cross section $\langle\sigma v\rangle_{2}^{\mathrm{UL}}$ for each galaxy cluster (see Table 1).

The two smallest 95\% CL upper bounds of the annihilation cross section $\langle\sigma v\rangle_{2}^{\mathrm{UL}}$ are close to $\langle\sigma v\rangle \leq 9 \times 10^{-26} \mathrm{~cm}^{3} \mathrm{~s}^{-1}$ (A2877 and Fornax). Although this upper bound is somewhat greater than that obtained by using the data from the Galactic Center $\left(\langle\sigma v\rangle \leq 7.5 \times 10^{-26} \mathrm{~cm}^{3} \mathrm{~s}^{-1}\right)$, this is already the tightest upper bound ever obtained based on the data from galaxy clusters. This upper bound can be tightened to a greater extent if we could get a tighter upper bound on gamma-ray flux.

\section{Discussion}

Recently, it has been reported that the excess gamma-ray emission near the Galactic Center can be explained by the 30-40 GeV dark matter annihilation through $b \bar{b}$ channel. In this paper, we follow this model and use the Fermi-LAT data obtained from galaxy clusters to constrain the annihilation cross section. By considering the most recent cosmic ray model, the tightest 95\% CL upper limit on the cross section is $\langle\sigma v\rangle \leq 9 \times 10^{-26} \mathrm{~cm}^{3} \mathrm{~s}^{-1}$. If we can precisely model the cosmic ray contribution, the possible range of the annihilation cross section could be tightened to a smaller range.

On the other hand, it can be shown that the annihilation of dark matter particles can also provide the required energy source of soft and hard components of hot plasma in the Galactic Centre. The predicted cross section is $\langle\sigma v\rangle \approx(2-6) \times$ $10^{-26} \mathrm{~cm}^{3} \mathrm{~s}^{-1}$ [19]. All these results are generally consistent with each other and satisfy the constraints from cosmic microwave background and low-redshift data [20]. They are also close to the canonical thermal relic abundance cross section in cosmology $\langle\sigma v\rangle \approx 3 \times 10^{-26} \mathrm{~cm}^{3} \mathrm{~s}^{-1}$ [21].

Besides, recent analysis from a stack of the 79 richest nearby galaxy clusters obtained an upper luminosity limit of $\dot{N}_{\gamma}=1.7 \times 10^{44} \mathrm{ph} \mathrm{s}^{-1}$ per galaxy cluster in the $0.8-100 \mathrm{GeV}$ band [22]. In the sample we used, the galaxy cluster with the highest luminosity in the same energy band is A2244 with $\dot{N}_{\gamma}=7 \times 10^{43} \mathrm{ph} \mathrm{s}^{-1}$ for $\langle\sigma v\rangle \leq 9 \times 10^{-26} \mathrm{~cm}^{3} \mathrm{~s}^{-1}$. Therefore, our calculations still agree with the most recent analysis. Although the joint-likelihood analysis using a stack of galaxy cluster may be a better approach to study the dark matter annihilation, using nearby individual cluster can avoid systematic errors for distant galaxy clusters in the stack analysis. We can check the results from different approaches to ensure the constraints obtained are consistent with each other.

Moreover, we find that there are 2 important galaxy cluster candidates for the evaluation of dark matter annihilation. The gamma-ray annihilation flux is close to their corresponding upper limits obtained from the Fermi-LAT data. In particular, the data from Fornax can provide the best indicator on the dark matter annihilation besides the Milky Way. The advantage of using data from galaxy clusters is that we can neglect the contribution from pulsars, which is quite significant in Milky Way. Therefore, further observations on these galaxy clusters can give an alternative way to study the properties of dark matter annihilation.

To conclude, our result provides a self-consistent picture and a tighter constraint on the annihilation dark matter model. The rest mass and the annihilation cross section could probably be verified by the Large Hadron Collider Experiment in the future.

\section{Conflict of Interests}

The author declares that there is no conflict of interests regarding the publication of this paper. 


\section{Acknowledgment}

This work is partially supported by a grant from the Hong Kong Institute of Education (Project no. RG57/2015-2016R).

\section{References}

[1] K. N. Abazajian, N. Canac, S. Horiuchi, and M. Kaplinghat, "Astrophysical and dark matter interpretations of extended gamma-ray emission from the Galactic Center," Physical Review D, vol. 90, no. 2, Article ID 023526, 2014.

[2] C. Gordon and O. Macias, "Dark matter and pulsar model constraints from Galactic Center Fermi-LAT gamma-ray observations," Physical Review D, vol. 88, Article ID 083521, 2013.

[3] D. Hooper, I. Cholis, T. Linden, J. M. Siegal-Gaskins, and T. R. Slatyer, "Millisecond pulsars cannot account for the inner Galaxy's GeV excess," Physical Review D, vol. 88, no. 8, Article ID 083009, 2013.

[4] R. Bartels, S. Krishnamurthy, and C. Weniger, "Strong support for themillisecond pulsar origin of the Galactic center $\mathrm{GeV}$ excess," http://arxiv.org/abs/1506.05104.

[5] S. K. Lee, M. Lisanti, B. R. Safdi, T. R. Slatyer, and W. Xue, "Evidence forunresolved gamma-ray point sources in the inner galaxy," http://arxiv.org/abs/1506.05124.

[6] T. Daylan, D. P. Finkbeiner, D. Hooper et al., "The characterization of the gamma-ray signal from thecentral milky way: a compelling case for annihilating dark matter," http://arxiv.org/abs/ 1402.6703 .

[7] E. Izaguirre, G. Krnjaic, and B. Shuve, "Bottom-up approach to the Galactic Center excess," Physical Review D, vol. 90, no. 5, Article ID 055002, 2014.

[8] F. Calore, I. Cholis, and C. Weniger, "Background model systematics for the Fermi GeV excess," Journal of Cosmology and Astroparticle Physics, vol. 2015, no. 03, article 038, 2015.

[9] B. Moore, T. Quinn, F. Governato, J. Stadel, and G. Lake, "Cold collapse and the core catastrophe," Monthly Notices of the Royal Astronomical Society, vol. 310, no. 4, pp. 1147-1152, 1999.

[10] J. F. Navarro, C. S. Frenk, and S. D. M. White, "A universal density profile from hierarchical clustering," The Astrophysical Journal, vol. 490, no. 2, pp. 493-508, 1997.

[11] M. Ackermann, "GeV $\gamma$-ray flux upper limits from clusters of galaxies," The Astrophysical Journal, vol. 717, no. 1, article L71, 2010.

[12] S. Ando and D. Nagai, "Fermi-LAT constraints on dark matter annihilation cross section from observations of the Fornax cluster," Journal of Cosmology and Astroparticle Physics, vol. 2012, no. 7, article 017, 2012.

[13] M. Ackermann, M. Ajello, A. Albert et al., "Search for cosmicray-induced gamma-ray emission in galaxy clusters," The Astrophysical Journal, vol. 787, no. 1, 18 pages, 2014.

[14] A. Pinzke and C. Pfrommer, "Simulating the $\gamma$-ray emission from galaxy clusters: a universal cosmic ray spectrum and spatial distribution," Monthly Notices of the Royal Astronomical Society, vol. 409, no. 2, pp. 449-480, 2010.

[15] A. Pinzke, C. Pfrommer, and L. Bergström, "Prospects of detecting gamma-ray emission from galaxy clusters: cosmic rays and dark matter annihilations," Physical Review D, vol. 84, no. 12, Article ID 123509, 2011.

[16] J. A. R. Cembranos, A. de la Cruz-Dombriz, A. Dobado, R. A. Lineros, and A. L. Maroto, "Photon spectra from WIMP annihilation," Physical Review D-Particles, Fields, Gravitation and Cosmology, vol. 83, no. 8, Article ID 083507, 2011.
[17] M. Schaller, C. S. Frenk, R. G. Bower et al., "Baryon effects on the internal structure of $\Lambda$ CDM haloes in the EAGLE simulations," Monthly Notices of the Royal Astronomical Society, vol. 451, no. 2, pp. 1247-1267, 2015.

[18] M. Ackermann, "Dark matter constraints from observations of 25 Milky Way satellite galaxies with the Fermi Large Area Telescope," Physical Review D, vol. 89, no. 4, Article ID 042001, 2014.

[19] M. H. Chan, "Indirect evidence of GeV dark matter," Monthly Notices of the Royal Astronomical Society Letters, vol. 448, no. 1, pp. L87-L89, 2015.

[20] M. S. Madhavacheril, N. Sehgal, and T. R. Slatyer, "Current dark matter annihilation constraints from $\mathrm{CMB}$ and low-redshift data," Physical Review D, vol. 89, no. 10, Article ID 103508, 2014.

[21] L. Lopez-Honorez, O. Mena, S. Palomares-Ruiz, and A. C. Vincent, "Constraints on dark matter annihilation from $\mathrm{CMB}$ observations before Planck," Journal of Cosmology and Astroparticle Physics, vol. 2013, no. 7, article 046, 2013.

[22] R. D. Griffin, X. Dai, and C. S. Kochanek, "New limits on gamma-ray emission from galaxy clusters," The Astrophysical Journal, vol. 795, article L21, 2014. 

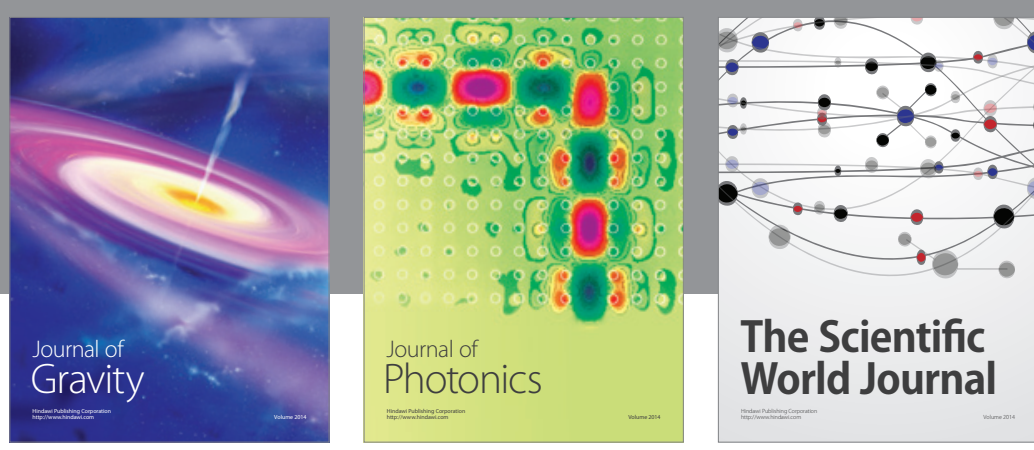

The Scientific World Journal
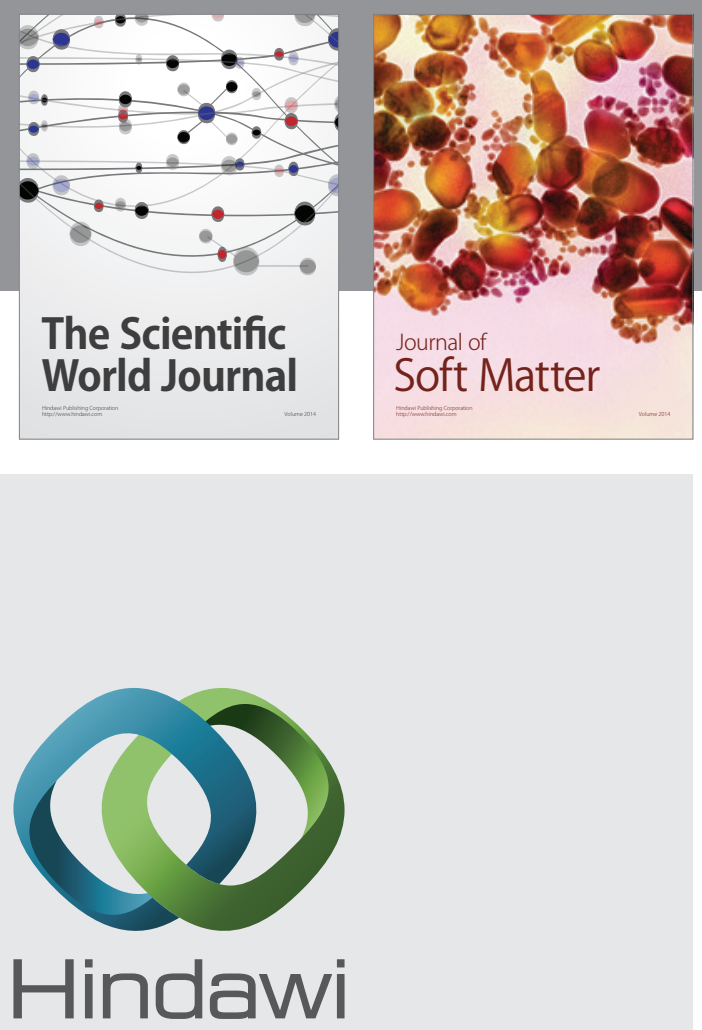

Submit your manuscripts at

http://www.hindawi.com

nternational Journal of

Statistical Mechanics
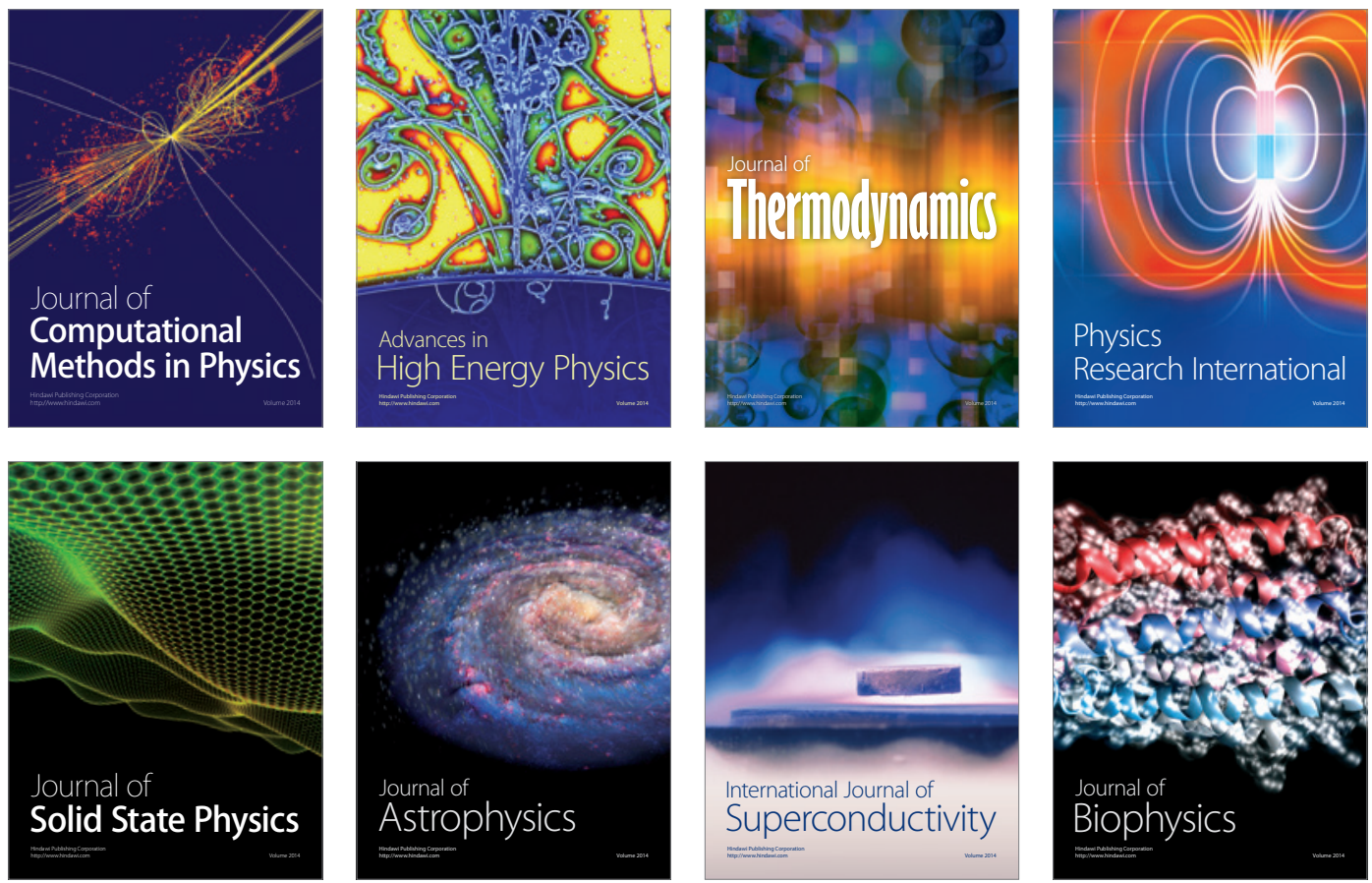
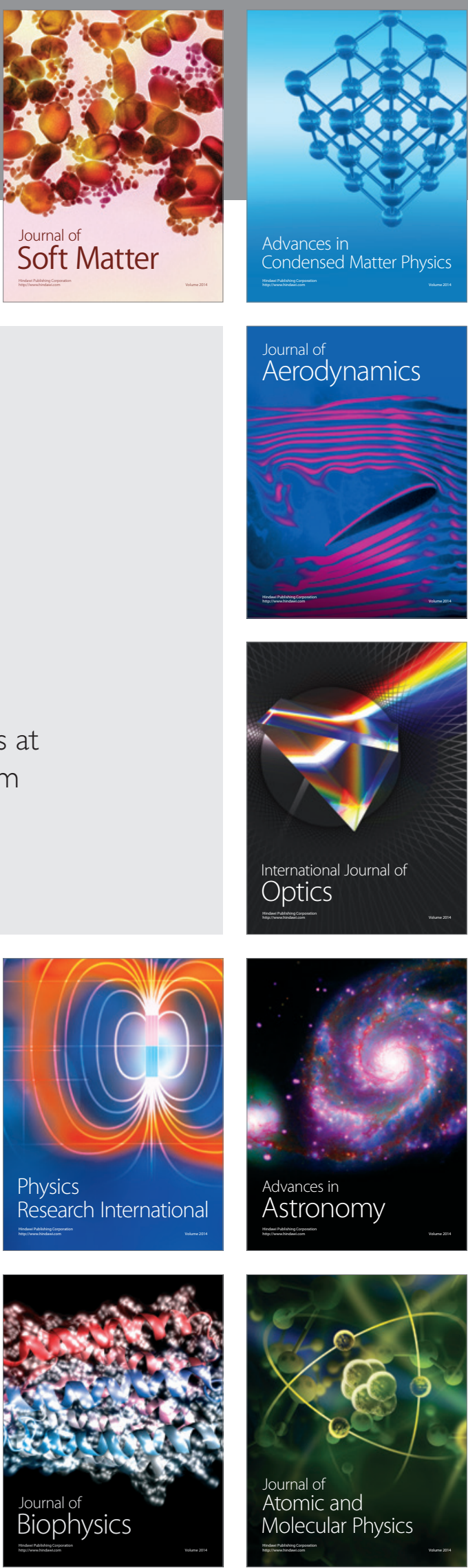